\title{
Monotonous and thousands-of-years cyclic components of isolated radio pulsars spindown
}

\author{
Anton Biryukov \\ Sternberg Astronomical Institute of MSU, Moscow, Russia \\ E-mail: eman@sai.msu.ru
}

\section{Grigory Beskin}

Special Astrophysical Observatory of RAS, Nizhnij Arkhyz, Russia

E-mail: beskinesao.ru

\section{Sergey Karpov}

Special Astrophysical Observatory of RAS, Nizhnij Arkhyz, Russia

E-mail: karpov@sao.ru

\begin{abstract}
In this article we revise the problem of anomalous values of pulsar' braking indices and frequency second derivatives arising in observations. The intrinsic evolutionary braking is buried deep under the superimposed non-evolutionary processes, that prevent to estimate directly its parameters for the majority of pulsars. We re-analyze the distribution of "ordinary" radio pulsars on a $\ddot{v}-\dot{v}$ and $n_{o b s}-\tau_{c h}$ diagrams assuming their spin-down to be a superposition of a "true" monotonous and a symmetric oscillational terms. We demonstrated that their influences may be clearly separated using simple ad-hoc arguments. Using maximum likelihood estimator we derived the parameters of both components and found the pulsar' peculiar evolution to be consistent with classical magnetodipolar law with braking index $n \approx 3$, while the oscillations are large enough to significantly vary the observed spin-down rate and completely dominate the second frequency derivatives. So, observed pulsar' characteristic ages (and similar estimators that dependend on the observed $\dot{v}$ ) are biased up to $0.5 \ldots 5$ times. This naturally resolves the discrepancy of characteristic and independently estimated physical ages of several objects, as well as explain very large, up to $10^{8}$ years, characteristic ages of some pulsars.

We suggest these oscillations to be connected with the long-term precession of NS around its magnetic axis, probably under the influence of anomalous braking torque.
\end{abstract}

25th Texas Symposium on Relativistic Astrophysics - TEXAS 2010

December 06-10, 2010

Heidelberg, Germany 


\section{Introduction}

Radiopulsars are purely variable sources of radiation with periods that evolve with time. The resulting phase of their light curve may be well described by an (infinite) Taylor series $\phi(t)=$ $\phi_{0}+v\left(t-t_{0}\right)+\frac{1}{2} \dot{v}\left(t-t_{0}\right)^{2}+\frac{1}{6} \ddot{v}\left(t-t_{0}\right)^{3}+\ldots$ dominated by the lower order terms. At the same time, the majority of published theoretical models on pulsar braking predict the spin-down described by a differential equation in from of power law $\dot{v}=-K v^{n}[17,3]$, where $n$ is the (constant) braking index and $K$ depend on the individual physical properties of pulsar. If $K=$ const then $n$ is always a simple combination of an instant frequency and two its derivatives $n_{o b s}=v \ddot{v} / \dot{v}^{2}$. This drives the determination of pulsar timing parameters an irreplaceable tool for studying the physics of pulsar spin-down. The canonical value $n=3$ is true for the vacuum magnetodipolar model, pulsar wind decreases this value to $n=1$, multipole magnetic field vice versa leads to $n \geq 5$ [17].

Several decades of very detailed timing of hundreds of pulsars [9, 2, 13-15] demonstrated, however, that the measured phases and, therefore, estimated spin-down parameters behind the frequency derivative are not consistent with ones expected for a power-law braking with a physically reasonable parameters. Phase (or $v, \dot{v}$ ) residuals after second-order fit display, generally very complex, irregular behaviour, which often not consistent with predominant influence of $\ddot{v}$ term (see e.g. Fig. 3 in [14]) even for intervals outside glitches (so-called "timing noise"). Moreover, the calculated braking indices are extremely high, up to $10^{6}$, and their values (as well as $\ddot{v}$ ) are even negative for nearly half of pulsars where such measurements have been performed. So individual measurement of braking index seems to be scanty for studies of pulsar spin-down.

Up to date there is no widely accepted model, which describes peculiarities in pulsars' observed spin-down. But there are no reasons to doubt that measured $\ddot{v}$ 's are really due to pulsars' or ISM physics and not just artifacts arised from somewhat incorrect observational and reduction procedures.

However, while secular spin-down estimation is failed for individual pulsar, it possible to study timing evolution in average through analysis of the ensemble of objects. Such analysis may help to distinguish and describe the components of pulsars' spin-down that drives secular and peculiar evolution correspondingly. Indeed, secular component is likely to be common for all pulsars, while statistical properties of the peculiar component may help to reveal it nature and, hence, bring new insights in the anomalous braking indices problem.

Therefore, in this work, we undertaken an analysed of 297 pulsars with published data on second frequency derivatives and demonstrated these values may be used to estimate the parameters of both secular (evolutionary, monotonous) and additional, peculiar, components of a pulsar spindown under a simple and reasonable assumptions. As a result we conclude that observed second derivatives measured over sufficiently large time spans do reflect, at least partially, some long timescale process, common to all pulsars. We described properties of this process and estimated parameters of secular spin-down in terms of canonical power-law.

\section{Statistical analysis of pulsars' timing parameters}

\subsection{The subset}

The set of pulsars under investigation in the current work is similar to the one used in our 


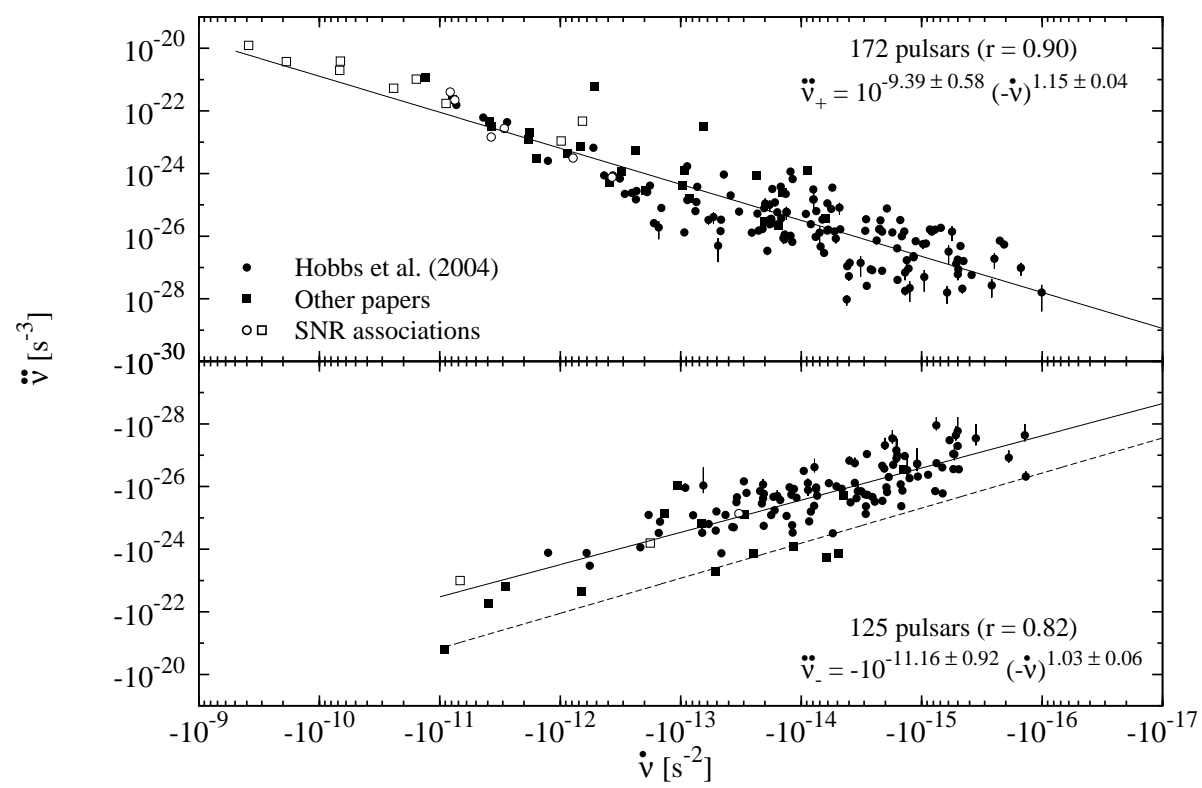

Figure 1: The $\ddot{v}-\dot{v}$ diagram for 297 pulsars. The figure shows the pulsars taken from [13] as circles, and the objects measured by other groups as squares. Open symbols represent the relatively young pulsars associated with supernova remnants. Analytical fits for both positive and negative branches are shown as solid lines. They were obtained using linear least-squares approximation in the logarithmic scale. Measurement errors are shown as error bars and in most cases are well inside the symbols. This diagram should be considered as an evolutionary one (pulsars systematically evolve from left to right of the plane) and may be explained with the idea of variations of pulsars' rotational parameters on a timescales that is significantly smaller than pulsar lifetime but, on the other hand, larger that observational time span [5]. The dashed line represents the approximation of the lower envelope of negative branch, estimated as a power-law by the method described in [7]. Its moduli may be considered a sensible estimation of the variational amplitude of $\ddot{v}$.

previous works $[4,5]$. From the 393 objects of the $\operatorname{ATNF}^{1}$ catalogue [16] with known $\ddot{v}$ we have compiled a list of "ordinary" radio pulsars with $P>20 \mathrm{~ms}, \dot{P}>10^{-17} \mathrm{~s} / \mathrm{s}$, and with relative accuracy of second derivative measurements better than $75 \%$. We have excluded recycled, anomalous and binary pulsars. 19 supplementary pulsars from other sources $[9,8]$ have been added. The final set consists of 297 objects including 247 from [13]. 18 of them are associated with young supernova remnants.

\subsection{Cyclic evolution of pulsars' spin-down}

Our work is based on the study of relations between quantities derived from pulsars' timing: $v, \dot{v}, \ddot{v}$, observed braking index $n_{o b s}=\ddot{v} v / \dot{v}^{2}$ and characteristic age $\tau_{c h}=-v / 2 \dot{v}$. The logarithms of $|\ddot{v}|$ show significant correlation with the ones of $-\dot{v}$ for both positive (172 pulsars, correlation coefficient $r \approx 0.9$ ) and negative (125 pulsars, $r \approx 0.82$ ) branches of the $\ddot{v}-\dot{v}$ diagram (Fig. 1). A similar correlations was also found in the $n_{o b s}-\tau_{c h}$ plot (Fig. 2).

Young pulsars there confidently associated with supernova remnants are systematically shifted to the left (open symbols on the both plots) and are absent on the right part of the diagrams. Hence,

\footnotetext{
${ }^{1}$ http://www.atnf.csiro.au/research/pulsar/psrcat/, revision from 6th Oct 2007
} 


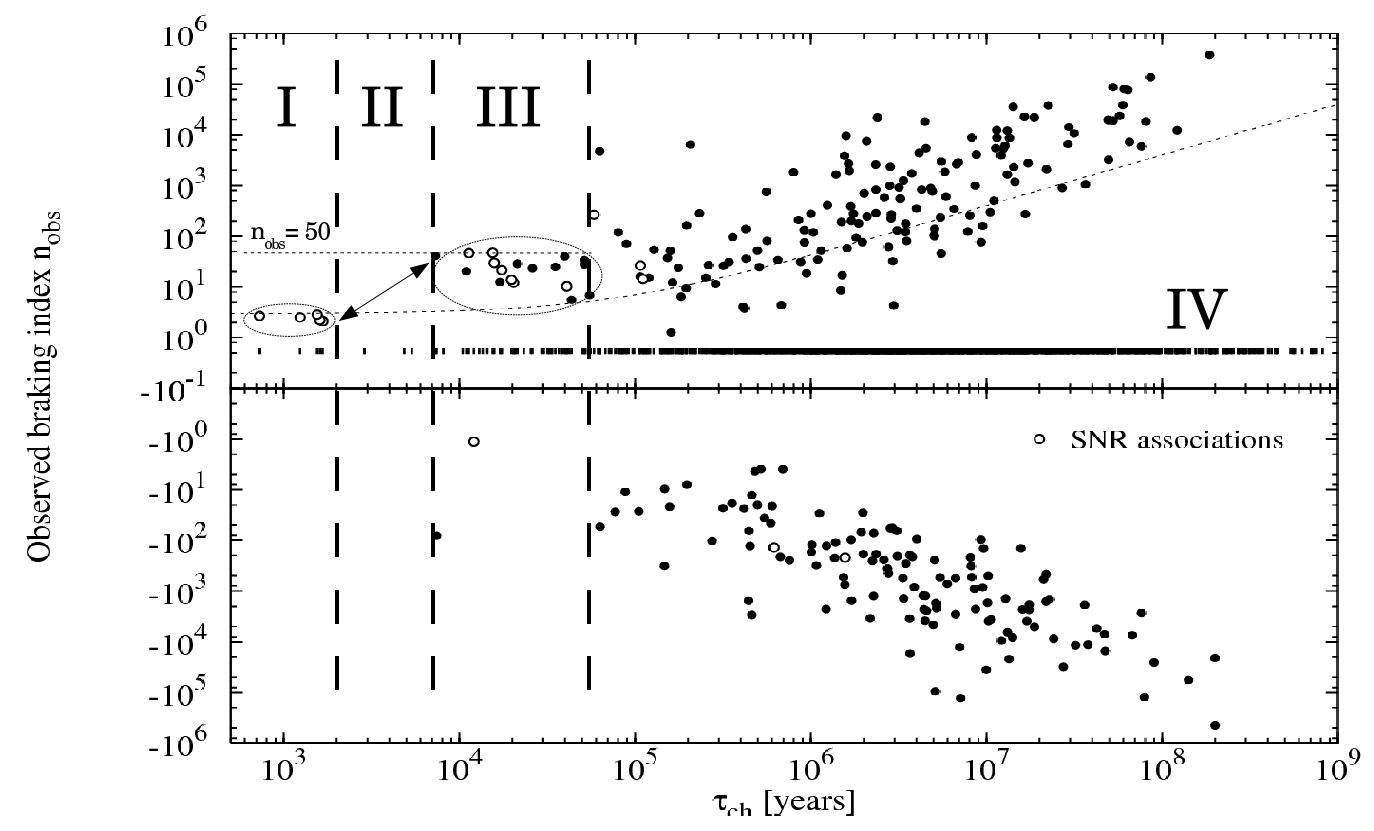

Figure 2: The $n_{o b s}-\tau_{c h}$ diagram. This diagram is similar to the $\ddot{v}-\dot{v}$ plot. The younger pulsars, partly associated with a SNRs, are located at the left part of the diagram (areas I, II and III). Their $\ddot{v}$ amplitudes are still quite small and are unable to produce negative observed $n$ even in area III. However, the braking indices of pulsars there are obviously anomalous $\left(n_{o b s} \sim 20 \ldots 50\right)$. At the same time idea of non constant coefficient in power-law spin-down can not explain these pulsars: the dashed line represents pulsar track with $K / \dot{K}=5 \times 10^{4}$ years, which is extremely short scale but it produces insufficiently small braking indices. All It means that their values are primarily affected by large $\dot{v}$ variations $(A \sim 0.5 \ldots 0.7)$. At the same time the lack of objects in area II jointly with small $n_{o b s}$ of pulsars of area I means that observed braking indices and ages of the latters are likely to be also affected by $\dot{v}$ variations. The observed $\tau_{c h}$ of the youngest pulsars are seems to be less than evolutionary values, while $\tau_{c h}$ of pulsars of area III vice versa are greater. The amplitudes of $\ddot{v}$ variations of pulsars of area IV are much greater than evolutionary values which produces two symmetric branches of the diagram. Short vertical lines along the $\tau_{c h}$ axes marks values of $\tau_{c h}$ of 1337 ordinary isolated pulsars with known $v$ and $\dot{v}$.

pulsars seems to evolve towards lower values of their $|\dot{v}|$ (higher $\tau_{c h}$ ). Note here that $\dot{v}$ and $\tau_{c h}$ are essentially highly correlated.

So, we conclude that, each pulsar during its evolution moves along the branches of $\ddot{v}-\dot{v}$ diagram while its $\dot{v}$ value increases. However, within the negative branch of $\ddot{v}-\dot{v}$, value of first derivative, being negative too, may only decrease with time, since $\ddot{v}$ is a formal derivative of $\dot{v}$, and both of them are regular components of observed rotational phase $\phi$. So, pulsar motion along there may be only backward, which clearly contradicts its evolutionary interpretation suggested earlier. The solution we offer is to assume the cyclic behaviour of pulsars on this diagram - as pulsar evolves, it repeatedly changes sign of $\ddot{v}$, in a spiral-like motion from branch to branch, and spends roughly half its lifetime on each one. So, we assuming the two-component structure of pulsars' spin-down $\dot{v}(t)=\dot{v}_{e v}(t)+\delta \dot{v}(t)=\dot{v}_{e v}(t)[1+\varepsilon(t)]$ where $\varepsilon(t) \equiv \delta \dot{v} / \dot{v}$ is a relative deviation, which is not necessarily small, but $|\varepsilon(t)|$ is likely less then unity $|\varepsilon(t)|<1$ due to the absence of "ordinary" pulsars with $\dot{v}>0$ in the subset under investigation. Also, value of $\dot{v}_{e v}(t)$ should be always negative for an isolated pulsar, which means secular loss of rotational energy, 
while $\ddot{v}_{e v}(t)$ is always positive.

The timescale of such variations has to be much shorter than the pulsar life time and at the same time significantly larger than the timescale of the observations to systematically affect the timing solution. Of course, the similar cyclic behaviour should be also inherent to other spin-down parameters $-v$ and $\dot{v}$. E.g. $\ddot{v}-\dot{v}$ diagram may be considered as a phase diagram of some cyclic process and, of course, pulsars will show similar behaviour on this diagram during all significant timing irregularities as timing noise and glitches. However, these processes are operates on the timescales that shorter than typical timespan length.

\subsection{Properties of the cyclic process}

The main characteristics of the cyclic, irregular component of $\ddot{v}$ behaviour - it amplitudes and timescales - may be derived from only deeper analysis of $\ddot{v}-\dot{v}$ and $n_{o b s}-\tau_{c h}$ relations with a simple, model-independent arguments. Amplitude $\mathscr{A}_{\dot{v}}$ of the second derivative variations is much greater than positive evolutionary trend $\ddot{v}_{e v}$ for the older pulsars and may be estimated with the lower envelope of the negative branch on $\ddot{v}-\dot{v}$ diagram (dashed line on the plot). At the same time amplitude of $\dot{v}$ variations $\mathscr{A}_{\dot{v}}$ is less then $\dot{v}_{e v}$ due to absence of pulsars with $\dot{v}>0$. After, assuming relation $\Omega \sim \mathscr{A}_{\dot{v}} / \mathscr{A}_{\dot{v}}$ we got $2 \pi / T=\Omega>\left(6.5 \times 10^{-11} \mathrm{rad} \cdot \mathrm{s}^{-1}\right) \cdot\left(-\dot{v}_{e v, 14}\right)^{0.12}$. It mean that $T_{u p} \sim 3 \times 10^{3}$ years and changes weakly from 1 to 5 thousands of years with pulsars' age. The corresponding amplitudes of $v$ variations, therefore, is much less then typical observed frequencies: $\delta v(t) \ll v_{e v}(t)$.

At the same time, after analysis of the group of young pulsars on the $n_{o b s}-\tau_{c h}$ diagram (in area III), the amplitudes of $\dot{v}$ variations may be estimated. We interpret these pulsars as an objects with high enough values of $|\varepsilon| \sim 0.5 \ldots 0.7$

Another important property of the cyclic process may be derived from an analysis of numbers of pulsars with positive and negative $\ddot{v}$. Indeed, these of pulsars $\left(N^{+}=172\right.$ and $\left.N^{-}=125\right)$ within the subset are significantly different. Assuming the probability $p=1 / 2$ for pulsar to have positive $\ddot{v}$, the hypothesis of an accidental nature of such a difference may be rejected by the binomial test on a $0.75 \%$ significance level. We investigated in detail behaviour of a significance levels for this binomial test and found that such asymmetry is significant only when relatively young pulsars are taken into account. Hence, since values of $\ddot{v}$ of older pulsars are mostly due to cyclic term $\delta \ddot{v}$, then $\delta \ddot{v}$ variations are symmetrical in respect to $\ddot{v}_{e v}$ and pulsars spends an equal amounts of time with $\ddot{v}$ greater and less of $\ddot{v}_{e v}$.

\section{Pulsars' spin-down model}

The symmetry of $\ddot{v}$ variations in respect to evolutionary term $\ddot{v}_{e v}$ may be useful for revealing of parameters of evolutionary part of spin-down, which is common for all pulsars. So, we defined the terms of the two-component model of observed pulsars' rotational evolution $\dot{v}=\dot{v}_{e v}(1+\varepsilon)$ as follows. The evolutionary term - according to the canonical power-law expression: $\dot{v}_{e v}=-K v_{e v}^{n}$ with constant $K$ and $n$; the cyclic term - as a simple harmonic relation $\varepsilon=A \cos \varphi$, where $A$ is a constant relative amplitude, and $\varphi(t)$ is variational phase.

Since intrinsic values of $\varepsilon$ are not observed, we simulated $A$ and $\varphi$ according to normal distribution $\mathscr{N}\left(\langle A\rangle, \sigma^{2}[A]\right)$ and uniform distribution within the $0 \ldots 2 \pi$ interval correspondingly. After, 


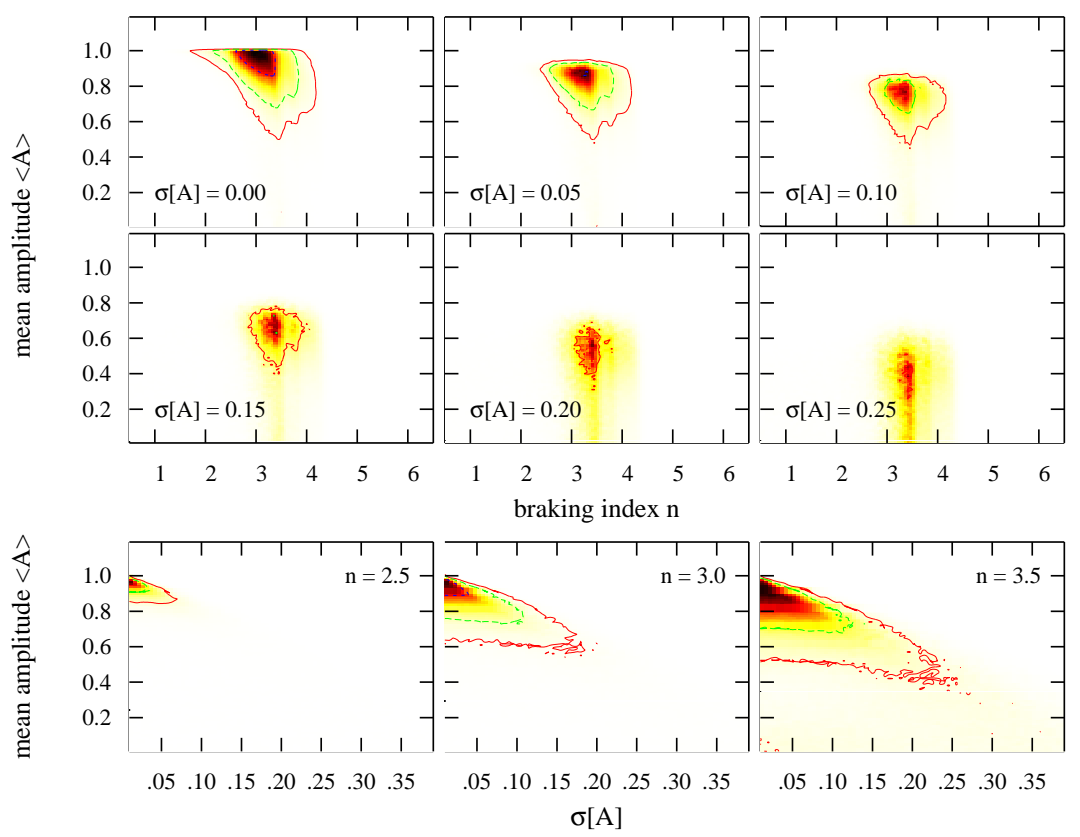

Figure 3: Plot of the confidence regions derived through the maximum likelihood estimator. The number of slices for fixed $\sigma[A]$ and $n$ are shown. Blue, green and red contours represent 65, 95 and $99 \%$ bounds. The values of evolutionary braking index $n$, mean amplitude $\langle A\rangle$ and its dispersion $\sigma[A]$, consistent with the symmetry of $\ddot{v}$ variations, are at $n \sim 2.5 \ldots 4,\langle A\rangle>0.5$ and $\sigma[A]<0.25$.

we calculated median value of coefficient $K: \hat{K}=\mathscr{M}\left[-\dot{v} / v^{n} /(1+\varepsilon)\right]$ and used it for $\ddot{v}_{e v}$ estimations. Since $\hat{K}$ dependent on the parameters of our model (n, $\langle A\rangle$ and $\sigma[A])$, then it is possible to variate their values and calculate corresponding number of pulsars with $\ddot{v}$ greater and less then $\ddot{v}_{e v}$. (The latter we estimated through differentiation of $\dot{v}_{e v}$ and using $\hat{K}$ instead of $K$ ). Assuming the symmetry of these numbers one can construct a criteria for best values of model parameters estimation. So, we used maximum likelihood criteria which we described in details in [6]. In the current paper we highlight our results only.

The contour maps of likelihood are shown in the Fig. 3 as a collection of slices of the parameter space for fixed $\sigma[A]$ (upper plots) or $n$ (lower plots). The 99\% confidence interval of evolutionary braking index covers the ranges $2.5<n<4$. The corresponding range of $\langle A\rangle$ depends on the accepted value of $\sigma[A]$. The decision about the appropriate amplitude and its dispersion can be made from analysis of $n_{o b s}-\tau_{c h}$ diagram provided above: high relative deviations $\varepsilon$ found from this analysis are consistent with high enough $\langle A\rangle$ and not so high $\sigma[A]$. So, we believe that the best choice is $0.5<\langle A\rangle<0.8$ and $\sigma[A] \sim 0.1$. Obtained value of $n$ is in a good agreement with the value $n=3$ expected from theory.

\section{Discussion and conclusions}

In the current work we analyzed the subset of 297 ordinary radiopulsars and conclude that there is exist a long-term mechanism, which operates on a timescales as long as thousands of years and which responsible for anomalous values of $\ddot{v}$ and braking indices $n_{o b s}$ arised from the observations. 
Such unusual timescale of long term variations - thousands of years - is much larger than the pulsar rotational period and, on the other hand, much shorter than its typical lifetime. At the same time, even the canonical magnetodipolar braking seems to already include an essential longterm variational mechanism: so-called anomalous braking torque due to near-field radiation will cause precession of NS around it magnetic axis with characteristic period of few thousands of years $[10,12,18]$. A similar effect may arising from the free precession of NS due to deformation along it magnetic axis [11]. However, simple precession is seems to be unable to explane so high amplitudes of $\ddot{v}$ variations that are observed. Hence some mechanism-mediator is needed. One possible such mechanism was offered in [1]. Ultimately, whether the monotonous precession of NS rotation axis around the magnetic moment able to produce any non-monotonous peculiarities in observed $\dot{v}$ and $\ddot{v}$ is not clear, but this is the only process suggested to operates on a such long timescales as thousands of years.

\section{Acknowledgments}

This work has been supported by the Russian Foundation for Basic Research (Grant No. 0402-17555), Russian Academy of Sciences (program "Evolution of Stars and Galaxies"), by the Russian Science Support Foundation and by the Grant of President of Russian Federation for the support of young Russian scientists (MK-4694.2009.2).

\section{References}

[1] D.P. Barsukov, A.I. Tsygan, MNRAS, 409 (2010) 1077

[2] A. Baykal, M. Ali Alpar, P.E. Boynton, et al., MNRAS 306 (1999) 207

[3] V. Beskin, A. Gurevich,\& Ya. Istomin, Physics of the Pulsar Magnetosphere, Cambridge University Press, Cambridge 1993.

[4] G. Beskin, A. Biryukov, S. Karpov, [astro-ph/0603375]

[5] A. Biryukov, G. Beskin, S. Karpov \& L. Chmyreva, AdSpR 40 (2007) 1498

[6] A. Biryukov, G. Beskin, S. Karpov, MNRAS submitted (2011)

[7] N. Cardiel, MNRAS 396 (2009) 680

[8] A.E. Chukwude, A\&A 406 (2003) 667

[9] F. D'Alessandro, P.M. McCulloch, E.A. King et al., MNRAS 261 (1993) 883

[10] L. Davis, M. Goldstein, ApJ 159 (1970) L81

[11] P. Goldreich, ApJ 160 (1970) L11

[12] M.L. Good \& K.K. Ng, ApJ 299 (1985) 706

[13] G. Hobbs, A.G. Lyne, M. Kramer et al., MNRAS 353 (2004) 1311

[14] G. Hobbs, A.G. Lyne, M. Kramer, MNRAS 402 (2010) 1027

[15] M.A. Livingstone, V.M. Kaspi, F.P. Gavriil, R.N. Manchester, ApJ 619 (2005) 1046

[16] R.N. Manchester, G.B. Hobbs, A. Teoh et al., AJ 129 (2005) 1993

[17] R.N. Manchester \& J.H. Taylor, Pulsars, Freeman, San Francisco 1977

[18] A. Melatos, MNRAS 313 (200) 217 